Provided for non-commercial research and education use. Not for reproduction, distribution or commercial use.

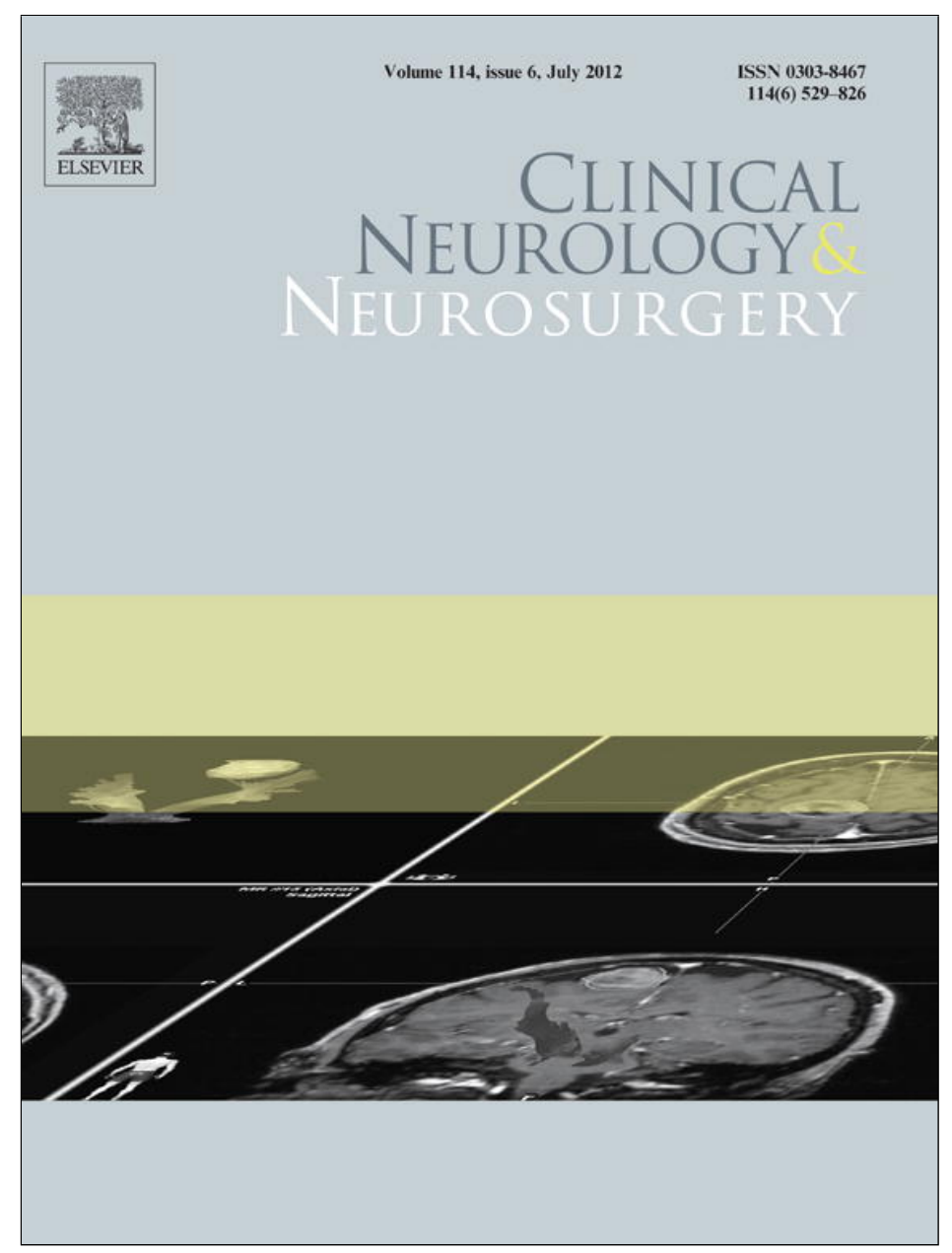

This article appeared in a journal published by Elsevier. The attached copy is furnished to the author for internal non-commercial research and education use, including for instruction at the authors institution and sharing with colleagues.

Other uses, including reproduction and distribution, or selling or licensing copies, or posting to personal, institutional or third party websites are prohibited.

In most cases authors are permitted to post their version of the article (e.g. in Word or Tex form) to their personal website or institutional repository. Authors requiring further information regarding Elsevier's archiving and manuscript policies are encouraged to visit:

http://www.elsevier.com/copyright 


\title{
Factors affecting the diagnostic delay in amyotrophic lateral sclerosis
}

\author{
Eleonora Cellura, Rossella Spataro, Alfonsa Claudia Taiello, Vincenzo La Bella* \\ ALS Clinical Research Center, Department of Experimental Biomedicine and Clinical Neurosciences, University of Palermo, via G La Loggia, 190129 Palermo, Italy
}

\section{A R T I C L E I N F O}

\section{Article history:}

Received 3 June 2011

Received in revised form

21 November 2011

Accepted 21 November 2011

Available online 14 December 2011

\section{Keywords:}

ALS

Diagnostic delay

Cognitive errors

\begin{abstract}
A B S T R A C T
Background: Although amyotrophic lateral sclerosis (ALS) is a relentlessly progressive disorder, early diagnosis allows a prompt start with the specific drug riluzole and an accurate palliative care planning. ALS at onset may however mimic several disorders, some of them treatable (e.g., multifocal motor neuropathy) or epidemiologically more frequent (e.g., cervical myelopathy).

Objective: To study the delay from onset to diagnosis in a cohort of ALS patients and to the variables that may affect it.

Methods: We performed a retrospective analysis of the diagnostic delays in a cohort of 260 patients affected by ALS $(M / F=1.32)$ followed at our tertiary referral ALS Center between 2000 and 2007.

Results: The median time from onset to diagnosis was 11 months (range: 6-21) for the whole ALS cohort, 10 months (range: $6-15)$ in bulbar-onset $(n=65)$ and 12 months (range: $7-23$ ) in spinal-onset $(n=195)$ patients $(p=0.3) .31 .1 \%$ of patients received other diagnoses before ALS and this led to a significant delay of the correct diagnosis in this group (other diagnoses before ALS, $n=81$ : median delay, 15 months [9.75-24.25] vs ALS, $n=179$, median delay, 9 months [6-15.25], $p<0.001$ ).

Conclusions: The diagnostic delay in ALS is about one year, besides the growing number of tertiary centres and the spread of information about the disease through media and internet. Cognitive errors based on an incorrect use of heuristics might represent an important contributing factor. Furthermore, the length of the differential diagnosis from other disorders and delays in referral to the neurologist seems to be positively associated with the delay in diagnosis.
\end{abstract}

(C) 2011 Elsevier B.V. All rights reserved.

\section{Introduction}

Amyotrophic lateral sclerosis (ALS) is a degenerative disease of the adult life, characterized by a progressive loss of spinal, bulbar and cortical motor neurons and, consequently, muscle wasting, dysphagia, dysarthria, and respiratory impairment. Death usually occur within 3-5 years after onset, the main cause being the respiratory failure [1]. Although the established pharmacological therapy for ALS, i.e. riluzole, allows an increase in survival [2], patients greatly benefit from the highly specialized palliative care, which is now best provided by tertiary referral Centres [3-5].

The diagnosis of ALS is mainly based on a clinical approach and supported by negative neuroimaging. Full nerve conduction studies and conventional EMG are very important in the diagnostic workup [6,7]. A recent consensus conference held by the Awaji Group established the equivalence of electrophysiological signs of lower motor

\footnotetext{
* Corresponding author at: ALS Clinical Research Center, Bio.Ne.C - via G La Loggia 1, 90129 Palermo, Italy. Tel.: +39 091 6555158; fax: +390916555162.

E-mail address: labella@unipa.it (V. La Bella).
}

neuron dysfunction to the clinical signs, making redundant the early category of "Laboratory-supported probable ALS" [7]. In addition, in the same conference it was established the equivalence, as a sign of acute denervation, between the fasciculation potentials and the fibrillation potentials and acute sharp waves [7]. Taken together, these two suggestions should increase the sensitivity of the diagnostic criteria and allow earlier diagnosis [7].

As ALS shows almost invariably a subtle onset, early diagnosis can be difficult in ALS, despite the growing number of clinical trials which undoubtedly encourage researchers to put much effort on a timely identification of the disorder. Previous studies have shown, in fact, little change in the diagnostic delay in ALS, with a median delay from onset to diagnosis of about 9-13 months [5,8-11].

The concept of "earlier is better" in the diagnosis of ALS bears significant implications in order to access to an appropriate management and care. Besides the available pharmacologic therapy, a high-quality and appropriate palliative care has been demonstrated to have a positive impact on the disease course, and hence on the patients' quality of life and survival [12-16].

The objective of the present study was to describe those factors that may delay the diagnosis in ALS and to discuss the potential for improvement. 


\section{Patients and methods}

Two-hundred and sixty clinically probable or definite ALS patients $(\mathrm{M} / \mathrm{F}=1.32)$ were diagnosed according to El-Escorial/WFN criteria [6] and followed by our Tertiary Referral Centre during a seven-years period (2000-2007).

We made a complete diagnostic work-up in 97 patients (37.3\%), while the remaining 163 patients (62.7\%) were referred to us from other Centres/Hospitals. All patients underwent a complete clinical evaluation, EMG and nerve conduction studies, brain and/or cervical MRI (the imaging studies were performed in over $85 \%$ of patients), and an extensive biochemical workup. This included a lumbar puncture in over $80 \%$ of patients.

One-hundred and ninety-five patients were spinal-onset (75\%), whereas in sixty-five patients the onset was bulbar (25\%). Twohundred and fifty patients (96.1\%) were regularly taking riluzole $(100 \mathrm{mg} /$ day). The median age (years) and interquartile range (IQR) of the ALS patients at onset was 60 (51-67).

Each patient, either during the diagnostic work-up at our ALS Centre or when referred by other Hospitals/Centres, underwent a detailed interview on when and how the symptoms began, and the type of diagnostic work-up and specialists who made the first visits. Dysarthria was the most frequent first symptom complained by bulbar-onset ALS patients, followed by dysphagia and pseudobulbar manifestation. Muscular atrophy, weakness and, less frequently, fasciculations in one or more limbs were the common initial complaint of spinal-onset patients. Gait disturbance were less frequently reported at onset by this group of patients. In a few cases disease apparently started with a progressive respiratory failure or with cognitive changes. Furthermore, it was noted whether other diagnoses were made before ALS. The interview was not formally structured, however we took care to go through all the following points: (i) when the patients noticed the first symptoms (the month), and what symptom caught his/her attention and led to seeking medical help; (ii) when was the first specialist referral made and what was the first diagnosis; (iii) when was the first neurologist referral made; iv) when the correct diagnosis was made.

All data concerning the 260 patients followed were stored in a database (Access, Microsoft ${ }^{\circledR}$ ) and the corresponding medical records reviewed. We could therefore establish: (a) the delay from onset to the correct diagnosis; (b) how many specialists were sought with particular reference to the first neurologist; (c) what diagnosis was made before the diagnosis of ALS, and (d) how much an initially wrong diagnosis delayed the correct one.

We compared different groups of patients: men and women, patients at different ages, patients with bulbar and spinal symptoms at the beginning of the disease.

All analyses were done using SIGMASTAT 3.5 software package (Systat Software Inc., San José, CA, USA). The main demographic and clinical characteristics of the ALS patients were analysed using medians with interquartile ranges (IQ) and differences between groups assessed with Mann-Whitney's ranks sum test. All correlations were studied using Spearman's rank correlation coefficient. A Multivariate logistic regression analysis was performed to assess which variables are likely to be associated with initial misdiagnosis. Dichotomous independent variables were gender, site of onset and neurologist as first physician seen; continuous independent variables were age at onset and the diagnostic delay. $p$ values $<0.05$ were considered significant.

Our Institutional Ethic Committee reviewed and approved this study.

\section{Results}

Table 1 shows the demographic and clinical characteristics of the 260 ALS patients included in this study. 148 (57\%) were men and
Table 1

Demographic and clinical characteristics of the ALS patients.

\begin{tabular}{lll}
\hline & ALS & $p$ \\
\hline Age at onset (years), median [IQ range] & & \\
All $(n=260)$ & $60.0[51-67]$ & \\
Bulbar-onset $(n=65,25 \%)$ & $63.0[54-70]$ & $0.018^{\mathrm{a}}$ \\
Spinal-onset $(n=195,75 \%)$ & $59.0[50-66]$ & \\
M/F & & \\
All $(n=260)$ & 1.32 & \\
Bulbar-onset $(n=65)$ & 0.80 & \\
Spinal-onset $(n=195)$ & 1.56 & \\
\hline
\end{tabular}

${ }^{\text {a }}$ Mann-Whitney's rank sum test.

$112(43 \%)$ were women, with a M/F ratio of 1.32 . Patients were stratified by site of disease onset: 65 were bulbar $(25 \%, M / F=0.8)$ and 195 were spinal $(75 \%, M / F=1.56)$. Dysphagia and dysarthria were almost invariably the symptoms at onset in the bulbar patients. Among the spinal-onset group, first symptoms (e.g., weakness, fasciculations) were almost equally distributed between upper limbs (UL) and lower limbs (LL): onset UL, $n=91$ (46.5\%) vs onset LL, $n=103$ (53\%). In one patient onset was with respiratory deficit (0.5\%).

The median age at onset (years) was 60 [IQ range: 51-67] for the entire cohort; the bulbar-onset group was however older than the spinal-onset group (bulbar, 63 [IQ range: 54-70] vs spinal, 59 [IQ range: 50-66], $p=0.018$, Mann-Whitney's rank sum test).

\subsection{The diagnostic delay in ALS}

As outlined above (see Section 2), onset of the disease was established from the patient's history (i.e., month when first symptom appeared). The month of diagnosis was instead that in which a first written report with "ALS or motor neuron disease" was given to the patient. At the time of diagnosis, a clinical categorization according to El-Escorial was performed (6). In all patients of our cohort, a complete EMG/nerve conduction study was available before the first diagnosis of ALS/motor neuron disease was formally raised. This underscore the importance of the neurophysiological studies on the diagnostic process of ALS.

The median time from clinical onset to diagnosis in the whole cohort was 11 months [IQ range: 6-21]. In the bulbar-onset patients the delay appeared slightly shorter (median: 10 months [IQ range: 6-15]), whereas we found the opposite in the spinal-onset group (median: 12 months [IQ range: 7-23]. The difference was however not significant ( $p=0.25$, Mann-Whitney's rank sum test), even when groups were stratified by sex (data not shown). Median time to diagnosis was 10 months [IQ range: 6-21] for men and 11 months [IQ range: $6-19]$ for women $(p=0.707)$.

As shown in Fig. 1, after symptoms onset, it took a median time of three months [IQ range: $1-7]$ to see a doctor, and six months [IQ range: 3-12] to be observed by a second physician. The latter in the vast majority of cases (over 90\%) was a neurologist. Note that bulbar-onset patients sought for a physician earlier than those spinal-onset (bulbar, 2 months [IQ range: $1-5.5$ ] vs Spinal, 3 months [IQ range: $1-8.25$ ], $p=0.02$, Mann-Whitney's rank sum test). The elapsed time from first to second visit was instead no significantly different between bulbar-onset and spinal-onset patients (bulbar, median 6 months [IQ range: 4-11] vs Spinal, median 7 months [IQ range: 3-12], $p=0.83$ ). Therefore, while bulbar-onset patients are looking for a physician slightly earlier than those spinal-onset, a neurologist (who almost invariably was the second physician sought) is seen only after several months, irrespective of the site of onset.

We then investigated which physician was likely to be first seen according to the site of onset and asked whether this may have some impact on the diagnostic delay. Most bulbar ALS consulted 


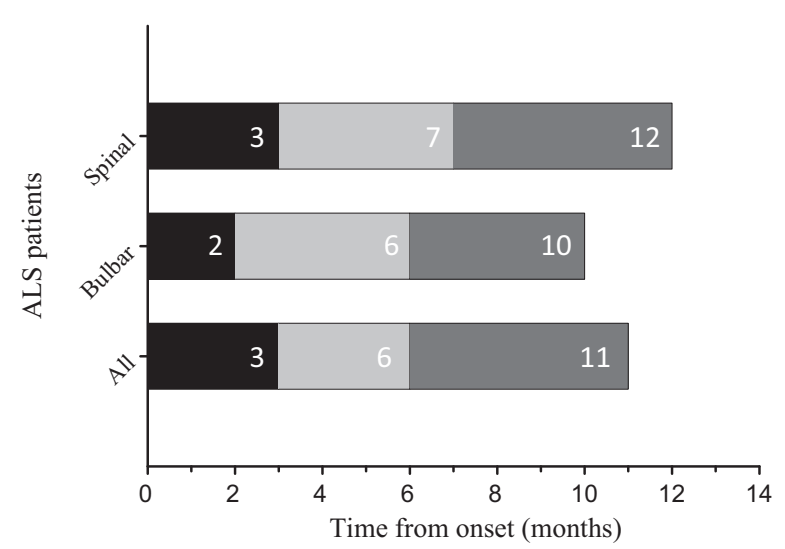

Fig. 1. Median elapsed time (months) between first symptoms leading to ALS and physician consultation. The horizontal bars indicate the whole ALS cohort (all, $n=260$ ), the bulbar-onset (bulbar, $n=65$ ) and the spinal-onset (spinal, $n=195$ ) subgroups. Black bars indicate the elapsed time between onset (T0) and the appearance of first symptoms. Bulbar-onset patients consulted a physician earlier than the spinal-onset patients ( $p=0.02$, time from onset to first physician consulted by bulbar-onset patients vs spinal onset, Mann-Whitney's rank sum test). Grey bars indicate the timing between first physician consultation and second physician consultation ( $p<0.001$, first physician vs second physician, Mann-Whitney's rank sum test). Note that in the vast majority of cases (over $90 \%$ ) the second physician was a neurologist. Dark grey bars show the timing between the second physician consultation and the final diagnosis.

an otolaryngologist (35\%), followed by a general practitioner (GP, $31 \%$ ) and a neurologist (26\%); almost half of the spinal ALS first sought for a neurologist (49\%), followed by a GP (24\%) and an orthopaedics (16\%). In summary, a neurologist was the first physician to be sought by 113 patients with a median delay of five months after onset [IQ range: 2-12], whereas the remaining 147 were first visited by another physician/specialist only 2 months after onset [IQ range: $1-5], p<0.001$ rank sum test. The difference was however completely lost when the final diagnosis was reached (median diagnostic delay in patients first seen by: (i) a neurologist, 11 months [IQ range: 6-18] vs (ii) another physician/specialist, 11 months [IQ range: $6-24], p=0.97)$. This suggests that the diagnostic pathway to ALS is a complex process that is not shortened when a patient is first seen by a neurologist.

Almost one-third of the patients in our cohort received other diagnoses (31.1\%, $n=81$ ) before the correct one, and in $36(44.4 \%)$ the initial misdiagnosis was made by a neurologist. Receiving an incorrect diagnosis led to a significant delay in reaching the right one in this group (other diagnoses before ALS, $n=81$ : median delay, 15 months [IQ range: 9.75-24.25] vs first diagnosis as ALS, $n=179$, median delay, 9 months [IQ range: 6-15.25], $p<0.001$, Mann-Whitney's rank sum test). A similar outcome was seen when the patients were grouped according to the site of onset (bulbar-onset: other diagnoses before ALS, $n=24$ : median delay, 12 months [IQ range: 8-16.5] vs first diagnosis as ALS, $n=41$, median delay, 7 months [IQ range: $5-15$ ], $p=0.08$, Mann-Whitney's rank sum test; Spinal-onset: other diagnoses before ALS, $n=57$ : median delay, 17 months [IQ range: 11.75-29.5] vs first diagnosis as ALS, $n=41$, median delay, 10 months [IQ range: 6-16.5], $p<0.001$, Mann-Whitney's rank sum test). The type of symptoms at onset and the specific regional location did not significantly affect the initial misdiagnosis (data not shown).

The most common misdiagnoses are listed in Table 2. The majority of initial wrong diagnoses were discal hernia and cervical myelopathy (32\%) or vascular pseudobulbar palsy (20\%), with little difference between neurologists and non-neurologist physicians.

We then assessed which variable is likely to be associated with the risk of misdiagnosis. As shown in Table 3, a multivariate logistic analysis suggested that the spinal onset of the disease correlates
Table 2

Most common misdiagnoses at onset in ALS.

\begin{tabular}{|c|c|c|c|}
\hline $\begin{array}{l}\text { Diagnoses } \\
(n=81)\end{array}$ & $\begin{array}{l}\text { Overall } \\
n(\%)\end{array}$ & $\begin{array}{l}\text { By a physician } \\
n(\%)^{\mathrm{a}}\end{array}$ & $\begin{array}{l}\text { By a neurologist } \\
n(\%)^{\mathrm{a}}\end{array}$ \\
\hline Arthrosis & $2(2.4)$ & $1(50)$ & $1(50)$ \\
\hline Alzheimer dementia ${ }^{\mathrm{b}}$ & $4(5.0)$ & $3(75)$ & $1(25)$ \\
\hline Depression & $5(6.2)$ & $3(60)$ & $2(40)$ \\
\hline $\begin{array}{l}\text { Discal hernia/cervical } \\
\text { myelopathy }\end{array}$ & $26(32.0)$ & $12(46)$ & $14(54)$ \\
\hline $\begin{array}{l}\text { Vascular pseudobulbar } \\
\text { palsy }\end{array}$ & $16(20.0)$ & $12(75)$ & $4(25)$ \\
\hline Myasthenia & $6(7.4)$ & $3(50)$ & $3(50)$ \\
\hline Neuropathy/myopathy & $7(8.6)$ & $4(57)$ & $3(43)$ \\
\hline Parkinson disease & $4(5.0)$ & $2(50)$ & $2(50)$ \\
\hline Thyroid dysfunction & $2(2.4)$ & $2(100)$ & $0(0)$ \\
\hline Carpal tunnel syndrome & $5(6.2)$ & $3(60)$ & $2(40)$ \\
\hline Multiple sclerosis & $2(2.4)$ & $0(0)$ & $2(100)$ \\
\hline Stroke & $1(1.2)$ & $0(0)$ & $1(100)$ \\
\hline Essential tremor & $1(1.2)$ & $0(0)$ & $1(100)$ \\
\hline
\end{tabular}

a Percent value in relationship to the number of diagnoses in the specific category.

b Two of the patients initially diagnosed by neurologists as having Alzheimer dementia.

positively with a higher risk of misdiagnosis. Being first visited by a neurologist conferred instead a slight lower risk of misdiagnosis. In both cases, however, the associations were not significant.

\section{Discussion}

This retrospective study involved a cohort of 260 ALS patients followed in a single tertiary referral centre, and it showed that the diagnostic delay is less than one year being slightly shorter in bulbar-onset patients. This appears to be similar to the findings of previous studies [5,8-11,13,17-20], and it is in part related to misdiagnoses, the most frequent being discal hernia/cervical myelopathy and vascular pseudobulbar palsy. Furthermore, an early consultation with a neurologist does not seem to shorten the diagnostic delay.

Several years after the establishment of the clinical criteria for the diagnosis of ALS [6] and the growing public awareness about the disease, the diagnostic delay does not appear to have been shortened despite the fact that most patients have free access to the Internet and/or they refer to a physician relatively early after symptoms onset and a neurologist is growingly often sought. This might in part be related to the putative negative impact of physicianrelated cognitive and psychological variables [8].

Several studies have focused on the diagnostic delay in ALS. The delay have been reported to range between eight and more than fifteen months (Table 4), with little improvement over the last years $[8,11,13,18-21]$, even after the creation of tertiary multidisciplinary ALS centres and prospective surveillance registries $[4,5,9,10,17,22]$. The delay is never less than eight months, although patients usually refer to a physician some three months after symptoms onset. The median delay from disease onset to diagnosis was calculated from the studies shown in Table 4, most of them carried out in the last two decades, and showed to be 11.7 months. We found a strikingly similar delay, even though a potential drawback of the present study is that it is based on a cohort from a tertiary referral centre. The availability of detailed clinical criteria [6] and

Table 3

Multivariate analysis of misdiagnosis risk in the ALS cohort.

\begin{tabular}{llll}
\hline Independent variable & Odds ratio & $95 \% \mathrm{CI}$ & $p$ \\
\hline Male gender & 0.97 & $0.56-1.68$ & 0.91 \\
Age at onset & 1.00 & $0.92-1.03$ & 0.62 \\
Diagnostic delay & 1.02 & $1.00-1.03$ & 0.017 \\
Spinal onset & 1.54 & $0.82-2.88$ & 0.17 \\
Neurologist as first physician seen & 0.86 & $0.49-1.50$ & 0.61 \\
\hline
\end{tabular}


Table 4

Summary of different studies on the diagnostic delay from onset to diagnosis in amyotrophic lateral sclerosis.

\begin{tabular}{llcl}
\hline Study & Period & Delay (months) & Ref. \\
\hline Czaplinski et al. (2006) & $1984-1999$ & 12.99 & {$[13]$} \\
& $1999-2004$ & 14.21 & {$[13]$} \\
Traynor et al. (2000) & $1993-1998$ & 8.0 & {$[17]$} \\
Chiò et al. (2002) & $1995-1996$ & 11.1 & {$[21]$} \\
Chiò et al. (2009) & $1995-2004$ & 10.4 & {$[10]$} \\
Belsh and Schiffmann (1996) & N.I. $^{\mathrm{a}}$ & 12.3 & {$[19]$} \\
Kraemer et al. (2010) & N.I. $^{\mathrm{a}}$ & 10.5 & {$[11]$} \\
Househam and Swash (2000) & $1995-1999$ & 13.0 & {$[8]$} \\
Zoccolella et al. (2006) & $1998-1999$ & 9.3 & {$[29]$} \\
Donaghy et al. (2008) & $2006^{\mathrm{b}}$ & 15.6 & {$[-]$} \\
Present study & $2000-2007$ & 11.0 & \\
\hline
\end{tabular}

a N.I., not indicated: the exact period is not shown in the study.

b Prevalent cases on 1st January 2006 from Northern Ireland Register. The median diagnostic delay calculated from all studies (the present study is not included) is 11.7 months [IQ range: 10.4-13.0].

the opportunity to perform an accurate laboratory, EMG studies and imaging workup do not seem to be of much help in shortening the diagnostic delay and cut off misdiagnoses. In particular, the frequency of initial misdiagnoses was 27\% [18], 44\% [11], 45\% [22] and $61 \%$ [8] in several studies. We found a $31.1 \%$ of initial misdiagnoses which brought about a longer delay to the right diagnosis (i.e., 15 months), a result similar to that reported by Househam and Swash [8]. $44.4 \%$ of initial misdiagnoses were made by a neurologist in our study. This result is slightly higher than that reported in a survey, where $28 \%$ of misdiagnoses were made by neurologists [22].

The diagnostic delay appeared to be only slightly shorter when the patient had a bulbar-onset (10 months), and we found no significant differences with respect to the spinal-onset group. The lack of a significant difference in the diagnostic delay between bulbarand spinal-onset patients was similarly reported by Chiò et al. [20], whereas in other studies the opposite was found [11,23]. The diagnostic delay in bulbar-onset patients however ranges between 8 and 10 months in the majority of reports. In conclusion, even though the patients in this subgroup generally refer earlier to a physician this does not allow them to receive a prompt diagnosis.

A question arises of why, besides the establishment of mainly clinically-based criteria for the diagnosis of ALS [6] and the spread of information about the disease, the diagnostic delay is still about one year, that is nearly one-third of the natural history of the disease [1]. Furthermore, being first observed by a neurologist does not seem to shorten the diagnostic delay.

ALS is a terrible disease, with no specific therapy able to arrest its progressive course. Raising a suspect of ALS and then breaking the news to the patient and relatives is a difficult task. Several papers have been devoted to this important step $[3,24,25]$ and there is general awareness that this is a key step in the diagnostic and management process. Recently, several works have been dedicated to cognitive errors that physicians make in their diagnostic work-up $[26,27]$. These may lead to missed diagnoses or mismanagement by neurologists [27]. Given that the logistic regression analysis showed initial misdiagnosis in ALS is unrelated to variables as sex, age at onset, diagnostic delay, site of onset, and being first observed by a neurologist, we suggest that cognitive errors, with particular reference to framing effects and anchoring heuristics [27], may at least in part play a relevant role in the diagnostic delay in ALS. The fact that most misdiagnoses of ALS (over 50\% in our study) are disk hernia/myelopathy and cerebrovascular disease supports this hypothesis. Symptoms and signs of ALS can in fact easily be framed in and anchored to these two common pathological conditions. Moreover, there are reports showing how much ALS patients undergo unnecessary surgery, peaking to more that $20 \%$ of cases $[11,28]$.
Even after careful correction for cognitive errors and a rigid application of the criteria for the diagnosis of ALS [6,27], we believe that a delay from onset to diagnosis in ALS cannot be reduced below three/four months for spinal-onset patients and one-two months for bulbar-onset patients.

An important variable that may greatly improve the diagnosis of ALS is the careful evaluation of the clinical progression of the symptoms. It is unlikely that hernia and vascular pseudobulbar palsy, which both account for by over $50 \%$ of all misdiagnoses, show a progression comparable to most ALS patients. In addition, a careful and extensive EMG and nerve conduction study is essential in shortening the diagnostic delay. In particular, the recent Awaji criteria [7] enable early detection of electrophysiological abnormalities in apparently unaffected limbs and outside the region of clinical onset [29]. Slowly progressing patients have to be followed up carefully and when only lower motoneuron signs and symptoms are apparent, common ALS-mimic disorders (e.g., Kennedy disease and [multifocal] motor neuropathy) have to be ruled out [30].

In conclusion, our study shows that the diagnostic delay in ALS is almost one year. Even with improved diagnostic tools, the establishment of careful clinical criteria and higher public awareness it appears to have undergone little variation over the years. Cognitive variables affecting the medical decision may play a role, and this seem to involve also neurologists, which partially contribute to a delayed diagnosis and misdiagnoses. A better clinical approach to the diagnosis, rather than mostly relying on laboratory tests and imaging techniques, may allow a significant reduction of the diagnostic delay in this devastating disorder.

\section{Acknowledgements}

This work was supported by a research grant of the Italian Ministry of Health (no. G71J07000010001). We thank Mr. Martin Russell for the revision of the English language.

\section{References}

[1] Spataro R, Lo Re M, Piccoli T, Piccoli F, La Bella V. Causes and place of death in Italian patients with amyotrophic lateral sclerosis. Acta Neurol Scand 2010;122:217-23.

[2] Orrell RW. Motor neuron disease: systematic reviews of treatments for ALS and SMA. Br Med Bull 2009;93:145-59.

[3] Miller RG, Jackson CE, Kasarskis EJ, England JD, Forshew D, Johnston W, et al. Practice parameter update: The care of the patient with amyotrophic lateral sclerosis: multidisciplinary care, symptom management, and cognitive/behavioral impairment (an evidence-based review). Neurology 2009;73:1227-33.

[4] Chiò A, Bottacchi E, Buffa C, Mutani R, Mora G. and the PARALS. Positive effects of tertiary centres for amyotrophic lateral sclerosis on outcome and use of hospital facilities. J Neurol Neurosurg Psychiatr 2006;77:948-50.

[5] Traynor BJ, Alexander M, Corr B, Frost E, Hardiman O. Effect of a multidisciplinary amyotrophic lateral sclerosis (ALS) clinic on ALS survival: a population-based study, 1996-2000. J Neurol Neurosurg Psych 2003; 74:1258-61.

[6] Brooks BR, Miller RG, Swash M, Munsat TL. El Escorial revisited: revised criteria for the diagnosis of amyotrophic lateral sclerosis. Amyotroph Lateral Scler Other Motor Neuron Disord 2000;1:293-9.

[7] de Carvalho M, Dengler R, Eisen A, England JD, Kaji R, Kimura J, et al. Electrodiagnostic criteria for diagnosis of ALS. Clin Neurophysiol 2008;119:497-503.

[8] Househam E, Swash M. Diagnostic delay in amyotrophic lateral sclerosis. What scope for improvement? J Neurol Sci 2000;180:76-81.

[9] Zoccolella S, Beghi E, Palagano G, Fraddosio A, Samarelli V, Lamberti P, et al. Predictors of delay in diagnosis and clinical trials entry of amyotrophic lateral sclerosis patients: a population-based study. J Neurol Sci 2006;250:45-9.

[10] Chiò A, Mora G, Calvo A, Mazzini L, Bottacci E, Mutani R. PARALS Epidemiology of ALS in Italy: a 10 year prospective population based study. Neurology 2009;72:725-31.

[11] Kraemer M, Buerger M, Berlit P. Diagnostic problems and delay of diagnosis in amyotrophic lateral sclerosis. Clin Neurol Neurosurg 2010;112:103-5.

[12] Oliver D. Palliative care. In: Oliver D, Borasio GD, Walsh D, editors. Palliative care in amyotrophic lateral sclerosis: from diagnosis to bereavement. Oxford University Press; 2006. p. 19-27.

[13] Czaplinski A, Yen AA, Simpson EP, Appel SH. Slower disease progression and prolonged survival in contemporary patients with amyotrophic lateral sclerosis 
Is the natural history of amyotrophic lateral sclerosis changing? Arch Neurol 2006;63:1139-43.

[14] Bourke SC, Tomlinson M, Williams TL, Bullock RE, Shaw PJ, Gibson GJ. Effect of non-invasive ventilation on survival and quality-of-life in patients with amyotrophic lateral sclerosis: a randomised controlled trial. Lancet Neurol 2006;5:140-7.

[15] Volanti P, Cibella F, Sarvà M, De Cicco D, Spanevello A, Mora G, et al. Predictors of on-invasive ventilation tolerance in amyotrophic lateral sclerosis. J Neuro Sci 2011;303:114-8.

[16] Spataro R, Ficano L, Piccoli F, La Bella V. Percutaneous endoscopic gastrostomy in amyotrophic lateral sclerosis: effect on survival. J Neurol Sci 2011;304:44-8.

[17] Traynor BJ, Codd MB, Corr B, Forde C, Frost E, Hardiman O. Clinical features of amyotrophic lateral sclerosis according to El-Escorial and Airlie House diagnostic criteria. A population-based study. Arch Neurol 2000;57:1171-6.

[18] Belsh JM, Schiffman PL. The amyotrophic lateral sclerosis (ALS) patient perspective o misdiagnosis and its repercussions. J Neurol Sci 1996;139:110-6.

[19] Pongratz D. The diagnostic process in ALS. J Neurol Sci 1999;165:S10-3.

[20] Chiò A, Mora G, Leone M, Mazzini L, Cocito D, Giordana MT, et al. Early symptom progression rate is related to ALS outcome. A prospective population-based study. Neurology 2002;59:99-103.

[21] Donaghy C, Dick A, Hardiman O, Patterson V. Timeliness of diagnosis in motor neuron disease: a population-based study. Ulster Med J 2008;77:18-21.
[22] Chiò A. ISIS survey: an international study on the diagnostic process and its implications in amyotrophic lateral sclerosis. J Neurol 1999;246(Suppl. 3):1-5.

[23] Turner M, Scaber J, Goodfellow JA, Lord ME, Mardsen R, Talbot K. The diagnostic pathway and prognosis in bulbar-onset amyotrophic lateral sclerosis. J Neurol Sci 2010;294:81-5

[24] Borasio GD, Sloan R, Pongratz DE. Breaking the news in amyotrophic lateral sclerosis. J Neurol Sci 1998;160(Suppl. 1):S127-33.

[25] McCluskey L, Casarett D, Siderowf A. Breaking the news: a survey of ALS patients and their caregivers. ALS 2004;5:131-5.

[26] Groopman J. How doctors think. Boston MA: Houghton Mifflin Company; 2007.

[27] Vickrey BG, Samuels MA, Ropper AH. How neurologists think. A cognitive psychology perspective on missed diagnoses. Ann Neurol 2010;67:425-33.

[28] Srinivasan J, Scala S, Jones HR, Saleh F, Russell J. Inappropriate surgeries resulting from misdiagnosis of early amyotrophic lateral sclerosis. Muscle Nerve 2006;34:359-60.

[29] Okita T, Nodera H, Shibuta Y, Nodera A, Asanuma K, Shimatani Y, et al. Can Awaji criteria earlier diagnosis than the revised El Escorial criteria. J Neurol Sci 2011;302:29-32.

[30] Traynor BJ, Codd MB, Corr B, Forde C, Frost E, Hardiman O. Amyotrophic lateral sclerosis mimic syndromes. A population-based study. Arch Neurol 2000;57:109-13. 